\title{
LA PERFORMANCE : UN LIEU D’ÉCHANGES ET DE CONTROVERSES
}

Julie Bawin et Pierre-Jean Foulon

Éditions Ligeia | « Ligeia »

2012/2 $\mathrm{N}^{\circ} 117-120$ | pages 87 à 88

ISSN 0989-6023

Article disponible en ligne à l'adresse :

https://www.cairn.info/revue-ligeia-2012-2-page-87.htm

Distribution électronique Cairn.info pour Éditions Ligeia.

(C) Éditions Ligeia. Tous droits réservés pour tous pays.

La reproduction ou représentation de cet article, notamment par photocopie, n'est autorisée que dans les limites des conditions générales d'utilisation du site ou, le cas échéant, des conditions générales de la licence souscrite par votre établissement. Toute autre reproduction ou représentation, en tout ou partie, sous quelque forme et de quelque manière que ce soit, est interdite sauf accord préalable et écrit de l'éditeur, en dehors des cas prévus par la législation en vigueur en France. Il est précisé que son stockage dans une base de données est également interdit. 


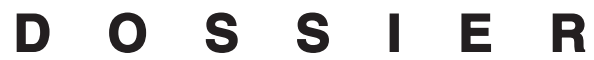

\section{LA PERFORMANCE : UN LIEU D'ÉCHANGES ET DE CONTROVERSES}

La performance constitue aujourd'hui l'une des manifestations les plus en vogue de la scène artistique internationale. Plusieurs événements, partout dans le monde, le prouvent. De festivals entièrement consacrés au genre - on pense notamment à la biennale Performa qui, depuis 2004, transforme la ville New York en happening géant - en passant par les expositions de musées, de galeries ou de centres d'art, la performance s'affiche telle une valeur ajoutée, devenant dans certains cas, et selon certaines circonstances, un mode de création aux allures parfois très institutionnelles. Apparue à la fin des années 50 sous l'impulsion d'artistes comme Allan Kaprow, John Cage et George Maciunas, la performance a longtemps été considérée comme un art de remise en cause de la matérialité et de la collectionnabilité de l'art. Ce que montrait la performance, autant que l'art conceptuel et d'autres propositions donnant la priorité à l'éphémère, c'était la fragilité de la notion d'œuvre d'art et la nécessité d'en élargir la définition et de décloisonner les disciplines. Non-spécialisation de l'artiste, processus ouvert, recours au hasard, déstabilisation des cadres normatifs, expérimentation, participation active du public, tels furent les fondements d'une pratique guidée par une relation très étroite entre l'art et la vie.

En dépit de son succès, la performance a ensuite été plutôt négligée, ne trouvant naturellement pas sa place sur le marché triomphant des années 80 . De cette situation naîtra non pas une disparition de ces pratiques, mais un élargissement considérable du champ d'action, donnant d'ailleurs lieu aujourd'hui à un retour remarqué, y compris dans le cadre muséal. La rétrospective consacrée récemment au Museum of Modern Art de New York à Marina Abramovic en témoigne, tout comme cette idée largement répandue dans le monde de l'art contemporain qu'il n'y a pas de bonne exposition sans performance. Les propositions actuelles ont par ailleurs ceci de particulier qu'elles n'appartiennent pas toutes au domaine artistique. D'où une extrême diversité des approches ; d'où, aussi, la difficulté pour l'historien ou le critique d'art d'en saisir les principales tendances, les axes majeurs. Malgré cette multiplicité difficilement palpable, il est néanmoins possible de dégager des lignes fortes, comme celle consistant, pour un nombre important de plasticiens, à rejouer ou à déjouer « les éléments supposés constitutifs de son histoire, comme ceux, supposés, de son ontologie (unicité, authenticité, vérité) $\rangle^{1}$. Tout un pan de la performance contemporaine s'affirme en effet comme le lieu privilégié d'une relation à son histoire. On épinglera une nouvelle fois l'exemple de Marina Abramovic qui, en 2003, fonde l'International Performance Group (IPG), un groupe réunissant une quinzaine de performers pratiquant de façon régulière des re-enactments de performances historiques, dont certaines ont été exécutées par Marina Abramovic elle-même ${ }^{2}$. Cette grande prêtresse de la performance n'est évidemment pas la seule à réactiver ou à renouveller les pratiques artistiques de la performance. Tino Sehgal, Francis Alÿs, Eric Duyckaerts, Michelangelo Pistoletto, Marika Buhrmann, pour n'en citer que quelque-uns, sont autant d'artistes qui nous montrent que la performance est un domaine de création en perpétuelle mutation.

NOTES

1. Goumarre, Laurent et Khim, Christophe, Performance contemporaine, in art press $2:$ Performances contemporaines, $\mathrm{n}^{\circ} 7$, novembre 2007-janvier 2008, p. 7.

2. Khim, Christophe, La performance à l'ère de son re-enactment, in Art-Press 2 : Performances contemporaines, $\mathrm{n}^{\circ}$ 7, novembre 2007 janvier 2008, p. 22. 
Au regard de l'étendue des questions que pose la performance, il est apparu nécessaire d'examiner ce mode de création comme un territoire à resituer, tant d'un point de vue historique que théorique. Le colloque «Art actuel et Performance $»^{3}$, dont les actes sont présentés dans le présent volume, a dès lors été pensé, non pas comme un état des lieux des formes récentes de la performance, mais comme une approche multiple de ses spécificités et des problématiques qu'elle soulève.

La question des origines, tout d'abord. Si d'aucuns considèrent la performance comme directement héritière des cérémonies initiatiques et des gestes rituels apparus dans les temps les plus reculés, certains, plus précis dans leurs analyses stylistiques et chronologiques, estiment que la performance s'enracine dans la radicalité des soirées futuristes et dadaïstes du début du XX $X^{\mathrm{e}}$ siècle. D'autres encore affirment que le début des performances coïncide plutôt avec la mise en question définitive, vers les années 60, des visions modernistes et, en particulier, de l'expressionnisme abstrait américain.

Définir la performance n'est guère plus aisé. Considérée comme une suite des prises de position en faveur de la dématérialisation de l'art et comme une conséquence de l'éclatement des catégories artistiques traditionnelles au sein d'un postmodernisme triomphant, la performance appartient tantôt à la carégorie des « happening », des « events », tantôt à celle du « Body Art » ou de l'art dit d'《 attitude ». Démêler les fils du nœud gordien que représente l'ensemble des pratiques artistiques qu'elle désigne est d'autant plus difficile que le problème de la délimitation du genre va de pair avec celui de la spécificité disciplinaire de la performance. Peut-on parler d'une pratique spécifique, alors même qu'elle se réfère à plusieurs displines (plastiques et théâtrales) et à différentes traditions (littéraires et musicales) ? Comme l'écrit Christophe Khim, il n'existe pas de réponse défintive, mais «l'on ne saurait se contenter d'une simple manœuvre pour retourner le problème en avançant cette instabilité définitionnelle comme propriété première de la performance $»^{4}$.

Si le problème de la définition divise, que faut-il penser des controverses suscitées par les problèmes de conservation et d'exposition d'un acte nécessairement lié au défilement du temps et donc, par nature, voué à l'effacement et à la disparition dans la dissipation de l'instant? La performance, très proche en cela des arts du spectacle, ne peut demeurer en mémoire que par le truchement de pratiques d'enregistrements sonores et visuels. La photographie et la vidéo sont donc étroitement associées à la performance dont elles tentent de conserver l'esprit, les conditions spectaculaires et certaines vertus d'instantanéité. Mais ces « traces » peuvent-elles êtres considérés comme des œuvres ? Ne s'agit-il pas plutôt de documents ou d'archives ? Quelle est leur part de justesse et de vérité ? Sont-elles reflet ou trahison ? Par ailleurs, qu'en est-il de leur dispersion éventuelle dans les arcanes du marché de l'art ? Transformée en denrée artistique et introduite bon gré mal gré dans les circuits de la marchandisation, la performance (ou son double virtuel) ne perd-elle pas son identité et sa force originelle?

Ces questions, qui elles aussi ne connaissent pas de réponses définitives, montrent combien il importe d'évaluer la performance à travers différentes prises de positions théoriques. N'aspirant ni à la dignité de l'inventaire, ni à l'héroïsme de l'exhaustivité, le colloque «Art actuel et Performance », dont les textes qui suivent rendent comptent, a donc été conçu comme un lieu d'échanges rassemblant des personnalités qui, par leurs parcours, leur expérience, leur pratique artistique ou leurs recherches, illustrent la diversité de réflexions dont la performance est aujourd'hui l'objet.

Julie Bawin et Pierre-Jean Foulon

3. Organisée par Charles Angelroth, Julie Bawin et Pierre-Jean Foulon, cette journée de colloque s'est tenue le 26 novembre 2011 aux Facultés Notre-Dame de la Paix à Namur.

4. Khim, Christophe, Une pragmatique de la performance, in art press 2 : Performances contemporaines 2, n ${ }^{\circ}$, août 2010 , p. 11. 